\title{
PID Controller Gains Tuning Using Metaheuristic Optimization Methods: A survey
}

\author{
Abdallah Abushawish ${ }^{1}$, Mohammed Hamadeh ${ }^{2}$, Ali Bou Nassif ${ }^{3}$ \\ University of Sharjah \\ Sharjah, UAE \\ U18105749@sharjah.ac.ae $e^{1}$, U18106063@sharjah.ac.ae ${ }^{2}$, anassif@sharjah.ac.ae ${ }^{3}$
}

\begin{abstract}
In nowadays industry, most processes are controlled and automated. Interestingly, PID controllers are major contributors to the control process since they were invented and become quite practical. PID controllers are vital component in the industry and enhancing the component will show an echo effect in today's technology. Their drawbacks are tuning them for an application, and this provides inspiration to develop advanced optimization methods in tuning PID controllers. This survey aims to review metaheuristic optimization methods of PID controller tuning that were published between 2010 and 2018. The paper was constructed based on 22 research papers and found that 8 metaheuristics optimization methods were used with PID tuning on 5 industrial applications. The papers also extensively provided answers to 3 research questions and assessing the quality of the papers based on 6 parameters.
\end{abstract}

Keywords: PID Controller, Tuning, Metaheuristic Algorithms, Optimization Techniques, Survey

\section{INTRODUCTION}

It is quite vital to control any application process, as it will reduce the consumption of resources and time, as well as avoiding unnecessary behavior from process. Therefore, the need to control a process gave inspiration to create and develop new tools capable of providing precise control. Additionally, the tools used to control have paved the way for other technologies to drastically improve. Now it is possible to manipulate the very large and quite small (Nanotechnology), bending and mending to produce structures of various sizes to serve an important purpose. Proportional, Integral and Derivative (PID) controller is a control tool heavily relied on in the industry of process control applications because of their practicality. These controllers are simple to design, implement and readjust, thus making them versatile. Once properly tuned for an application, a PID controller reduces errors, through controlling a process instability and improving response time showing great promise. Yet the process of tuning the PID controller could prove troublesome. Furthermore, even after tuning and providing a satisfactory behavior, that tuning setting might not be the optimal performance the controller could provide. In other words, the controller could be tuned even further [1], [2]. There are plenty of research studies conducted on PID tuning such as developing, improving, comparing and reviewing methods of tuning. Even with the great amount of research about PID tuning using metaheuristics optimization methods, there does not exist a proper fully comprehensive literature review [3], [4]. The inspiration of this survey is derived from the extended reach of our knowledge that, almost no review concentrates on metaheuristics optimization methods and systematically review by clearly identifying research questions on the topic.

This paper presents and illustrates the research done from $1^{\text {st }}$ of Jan 2010 till $31^{\text {st }}$ of Dec 2018. The paper objective is to gather, filter and assess the available papers in that time period. Additionally, the paper raises three research questions, and provides answers acquired from the statistical study performed on the gathered papers. Finally, the paper is split into six sections. Section 2 identifies and provides a quick discussion over some related works. Section 3 provides a generalized background on the topic. Section 4 elaborates on the methodology used to provide the systematic literature review. Section 5 displays the results and finding. Section 6 concludes the paper and discusses future works.

\section{RELATED WORK}

Several reviews have been presented in the topic of proportional, integral and derivative (PID) controller gain tuning methods. Bansal et al. [3] reviewed modern and classical methods for PID controller gain tuning and have classified them into two categories, the classical methods and optimization methods. Additionally, the review provided an analytical comparison between the various techniques such as the use of Ziegler Nichols method, Genetic Algorithm, Ant Colony Optimization, Artificial Neural Networks and others. Finally, the paper concludes that a detailed analytical study of all the PID tuning methods is yet to be performed.

Ghosal et al. [4] performed a review study on PID controller gains tuning that provide an efficient and promising performance using various swarm optimization methods. The paper presented an introductory literature review that utilize swarm intelligence methods like Bacterial Foraging Optimization (BFO), Particle Swarm Optimization (PSO), and Ant Colony Optimization (ACO). Additionally, demonstrated a comparative study that details the pros and contras of each optimization method. Finally, the paper concluded that the swarm optimization methods could be 
modified slightly for the application of PID controller gain tuning, in order to provide a better tuning in reduced time frame.

Lastly, Singh et al. [5] illustrated a review on the tuning of PID controller gain used in the application of controlling a DC motor speed using soft computing methods. The soft computing methods used in this paper are Genetic Algorithm, Particle Swarm Optimization and Fuzzy Logic Algorithm.

In this paper a systematic literature review will conducted on the topic of PID controller gain tuning. Some research questions have been raised and answered in the hopes that it might prove helpful as reference to any future technical research to be conducted. The paper focuses on the use of optimization methods used in the tuning of PID controller gains.

\section{BACKGROUND}

Industrial processes heavily rely on the control of any system behavior, and Proportional-Integral-derivative PID controllers are mostly used to control the response of plant. PID controllers are cost-friendly and are quite simple to design and implement to control and improve a plant response to meet the needs of the process efficiently. PID controller's capacity to control depends on gains to each part of the system, and if these gains are properly tuned, may provide the change needed in the plant. The response of the plant upon operation may overwork (overshot) or can be very unstable in reaching the desired performance. If a PID controller is finely tuned it will eliminate any undesired behavior the system might undergo, and therefore without tuning the gains the PID controller is quite useless [1], [2], [6]. It is clear that PID controllers must be tuned in order for PID to function as needed but the process of tuning isn't that obvious and requires different approaches and techniques to get the desired behavior out of the system. If a plant is modeled mathematically it is possible to tune the PID controller using Mathematical approach and derive the values that way. The issue is that not only is mathematics hard and requires solving problem in the frequency domain through Laplace transformations but also it might not provide the optin ' ${ }^{\prime}$ ' $\mathrm{s}$ that could be achieved. Another problem is that a sing (1) d PID controller might show great performance with a certain system but once applied on all other systems will generate undesired errors, thus PID controllers need to be tuned for each system separately [1], [2], [6]. The proportional, integral and derivative controllers work together to ensure the effective, smooth and stable response from the plant. The proportional controller ensures correcting present errors proportionally, meaning if the present error is larger the output signal from the controller is large and vice versa. The process of the proportional controller, if alone might drive the

$$
c(t)=K_{P} e(t)+K_{i} \int_{0}^{t} e(t) d t+K_{d} \frac{d}{d t} e(t)
$$

system unstable and always in the state of correcting the error. The integral controller sums up past errors to track the reference point and smoothly bring back the system to the reference point. The derivative controller responds largely to any big changes in the error signal, thus stabilizing huge proportional changes to avoid the shakiness and instability of the system behavior. Together these controllers work efficiently if their gains are finely tuned [1], [2], [6]. It is now obvious the tuning a PID controller isn't an easy task, but if to approach the problem from another perspective it might prove easier to tune. It is wise to model the system initially in the frequency domain, therefore the equation of the PID controller transforms using Laplace transformation and is shown below [1], [2], [6].

$$
C(s)=K_{p}+\frac{K_{i}}{s}+k_{d} s
$$

The transfer function of the plant in the frequency s-domain also effects the tuning of gains of the PID controller. There multiple techniques that tune PID controllers and are mainly categorized as

1. The Classical Methods

2. The Optimization Methods

\subsection{CLASSICAL METHODS}

Optimization methods have and are still being researched under different optimization algorithms [30]. The goal is to determine which algorithm performs the tuning process faster and with a better system response. Some of these methods are using Genetic Algorithm, Particle Swarm Optimization and another metaheuristic algorithm [3], [4], [5].

These methods are mainly formulated to minimize an error objective function and there four types of objective functions which are:

- Integral Absolute Error

$$
I A E=\int_{0}^{\tau}|e(t)|
$$

- Integral Square Error

$$
I S E=\int_{0}^{\tau}|e(t)|^{2}
$$

- Integral Time Absolute Error

$$
\text { ITAE }=\int_{0}^{\tau} t|e(t)|
$$

- Integral Time Square Error

$$
\text { ITSE }=\int_{0}^{\tau} t|e(t)|^{2}
$$

These optimization algorithms are metaheuristic in nature, and in other words run-on higher-level procedures to generate and update their heuristics in hopes of improving the autotuning of PID gains. All of them generally deal with a solution space of all possible values of the PID controller gains $K_{p}, K_{i}$ and $\mathrm{K}_{\mathrm{d}}$. In this case, the solution space is a three variable (3dimensional) since there are three gains. The algorithms run by generating random initial points and updating them in hope of reaching the optimal values of three gains that produce the least error index. 
In summary, the error index objective function depends on three dependences which are:

1. The system/plant and its behavior due to an input

2. The controller behavior and the tuned gains values

3. The input/desired response to the system

\section{METHODOLOGY}

The survey proposed is based on by Kitchenham and Charters' Systematic Literature Review (SLR) [7] and following the structure of [31]. This methodology consists of distinct steps and procedures which are planning phase, conducting phase, and reporting phase. Each of these phases has many stages. The first phase is divided into six stages, identifying the research questions, specifying the strategy of the research, identifying a proper selection of the study, specifying the rules for quality selection, designing the approach (strategy) for data extraction, and synthesizing the research's extracted data. The research questions were mainly based on the objectives of the review paper. In next stage, strategy was specified for retrieving the research papers related to the objective mentioned in first stage. Moreover, to get a precise search for research papers, the search terms and the article selection criteria were identified properly in this stage. Third stage was representing the proper study selection criteria which includes the rules we followed for the inclusion and exclusion of papers. The quality questions which were used for filtering the research papers provided in the fourth stage. In the fifth stage, the strategy for data extraction was described which was used to find proper answers for the raised research questions. The last stage was synthesizing the data extracted from the research papers to get the results. The review protocol which was followed in this review is illustrated in the following subsections.

\subsection{RESEARCH QUESTIONS}

The aim of this review is to identify the importance and the benefits of applying different metaheuristic algorithms to optimize the tuning of the proportional-integral-derivative controller (PID controller), and to find the most efficient algorithm. Based on that, we identify the following research questions:

- RQ1: What are the optimization techniques used in tuning PID gains?

The purpose of this question is to specify the different types of optimization techniques used in gain tuning of PID controller.

- RQ2: What are the domains (plants) which used the optimization techniques in PID tuning?

The aim of RQ2 is to identify the different plants which optimized using optimization techniques.

- RQ3: what is the most effective optimization algorithm used in tuning PID among the selected papers? why?
This question will provide us with the most efficient algorithm compared to other algorithms in gain tuning of the PID controller from our selected papers with reasonable reasons.

\subsection{SEARCH STRATEGY}

The search strategy which we implemented in this review is explained in detail as the following:

\subsubsection{SEARCH TERMS}

The procedure that we followed to conduct the search terms is as the following [7]:

1. The search terms were identified based on the research questions.

2. we specified new search term using the synonyms and the alternative spellings.

3. For the aim of limiting the results of the search, we used the Boolean operators (ANDs and ORs).

we used the double quotation (" ") for the exact phrases.

The search terms that we used in exploring and searching on the related publications are mentioned below:

- "PID" AND "tuning" AND "optimization".

- "PID tuning" AND "optimization".

- "PID" AND "tuning" AND "optimization" AND

"techniques" OR "technique".

- "PID controller" AND "optimization".

- "PID controllers" AND "optimization".

- "PID" AND "Gain" AND "optimization".

- "PID” AND “tuning” AND "Gain" AND “optimization".

\subsubsection{SURVEY RESOURCES}

We used the following online resources and digital libraries in searching for the required research papers:

- Google Scholar

- Science Direct

- IEEE Explorer

- Springer

- ACM Digital Library

\subsubsection{SEARCH PHASES}

The search terms previously specified were used to search for the research papers that are related to the topic from the specified digital libraries. Furthermore, going through the references of our selected papers allowed us to find the papers that answered the raised research questions. In next section, the inclusion and exclusion criteria are discussed in detail which were used to filter our papers. After applying the criteria of inclusion and exclusion, 22 articles were selected for the review paper.

\subsection{STUDY SELECTION}

From the first step of the search process, we found 200 papers originally. The process of selecting the relevant research papers were done individually. After that, we scheduled some 
meetings to make sure that we chose the most pertinent papers. The steps (shown in Fig.3) mentioned below describes the selection process followed in this review:

1) step 1: eliminating the identical papers from the online resources.

2) step 2: eliminating the review papers from the chosen papers as those papers were used in related work section to compare between this papers and other review papers.

3) step 3: applying the criteria of inclusion and exclusion on the selected papers.

4) step 4: applying the quality assessment rules which helps to find the most suitable answers for question raised.

The criteria of inclusion and exclusion is presented below: inclusion criteria:

- Include papers that mention the PID gain tuning.

- Include journal and conference papers only.

- Include papers which present optimization methods of PID gain tuning.

- Include papers that use metaheuristic methods for optimization.

- Include papers which compare between different metaheuristic methods.

- Include papers which compare between the proposed evolutionary method(s) and the conventional method(s).

- Include papers were published between 2010-2018.

Exclusion criteria:

- Exclude papers that mention PID but not related to the gain tuning.

- Exclude papers that use non-metaheuristic methods.

- Exclude papers that didn't use any optimization algorithm.

Finally, 22 papers were used in this review after applying the filtration procedures. The list of the selected papers is in Table 1 of the Appendix A.

\subsection{QUALITY ASSESMENT RULES (QARs)}

The final step to identify the selected papers is applying the quality assessment rules which help in evaluating the research papers and determining the suitability and strength of the papers according to our topic. we identify six QARs where each one has a value of 1 out 6 . The QAR score $=1$ if its fully answered, 0.75 if its above average, 0.5 if its average, 0.25 if its below average, and 0 if it's not answered. The gross score of each paper is the summation of the QARs' scores. The papers that score 3.5 or above were included; the others excluded.

1) QAR 1: Are the objectives of the research paper identified obviously?

2) QAR 2: Does the paper give enough background information about PID gain tuning?

3) QAR 3: Are the algorithms used clearly mentioned?
4) QAR 4: Is the proposed metaheuristic algorithm compared with other algorithms or with the conventional methods?

5) QAR 5: Are the problems that need to be optimized mentioned clearly?

6) QAR 6: Does the paper considered useful?

The scores of the selected papers are shown in the table 2 in the Appendix A.

\subsection{DATA EXTRACTION STRAREGY}

In this stage, we used the selected list of papers to extract the needed information that will help us in finding answers for the raised research questions. Thus, we tabulate the data needed in an extraction table (Table 1) [7]. Each one of us has extracted the information from 11 papers, where the total number of the selected papers was 22 papers. Then, meetings were conducted to discuss the results we got.

\begin{tabular}{|l|}
\multicolumn{1}{|c|}{ TABLE 1 } \\
EXTRACTION \\
\multicolumn{1}{|c|}{ DATA } \\
\hline Paper ID \\
Paper title \\
Publication year \\
Publication type \\
Domain \\
RQ1 \\
RQ2 \\
RQ3 \\
RQ4 \\
\hline
\end{tabular}

\subsection{SYNTHESIS OF EXTRACTION DATA}

The synthesis procedure in this review is as the following: In RQ1 and RQ2, we tabulated the extracted information using the method of narrative synthesis where we used the pie chart to demonstrate the percentages needed to answer those research questions. While, for RQ3 a quantitative procedure was used to find the best algorithm used for PID gain tuning.

\section{RESULTS AND DISCUSSION}

In this stage, the results we got from the review will be discussed. Each one of the research questions will be

\begin{tabular}{|c|c|c|}
\hline \multicolumn{3}{|c|}{ TABLE 2 } \\
\hline $\begin{array}{c}|c| \\
\text { THE QUALITY DISTRIBUTION } \\
(\%)\end{array}$ & Frequency & $\begin{array}{c}\text { Range of total } \\
\text { marks of QARs }\end{array}$ \\
\hline $41.7 \%$ & 25 & $0-1.5$ \\
$21.7 \%$ & 13 & $1.75-3.25$ \\
$13.3 \%$ & 8 & $3.5-4.5$ \\
$23.3 \%$ & 14 & $4.75-6$ \\
\hline $100 \%$ & 60 & The Total \\
\hline
\end{tabular}


answered in a separate subsection, so we will have 3 subsections in this stage in total. Those answers will be extracted from the 22 selected papers which implemented the optimization techniques in PID gain tuning. Moreover, the papers were streamed based on the quality assessment rules and the marks attained by each paper. The papers that got 3.5 marks out of 6 or above were considered.

Table 2 shows the frequency of the papers corresponding to a specified mark range. The 22 collected papers were used in this review categorized into conference papers and journals. The pie chart which represented in Figure 1 is demonstrating the percentages of papers corresponding to the type of each.

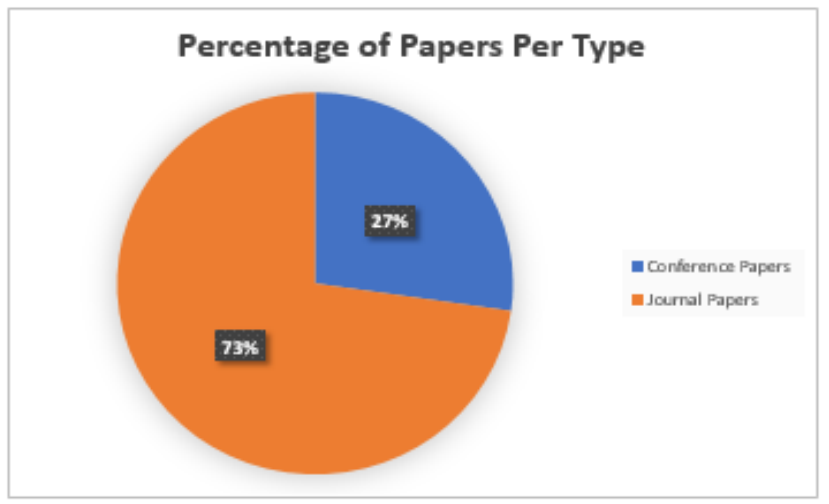

Figure 1- Pie Chart representing the percentage of each aper type.

It illustrates that the journal papers formed $73 \%$ of the total papers were used in this review. The rest $27 \%$ were identified as conference papers. Table 3 shows the distribution of conference papers which give more details about used papers. We can infer from the table 3 that there were 6 papers from six different conferences. The papers are equally distributed between EDT "Conference on E-Health Networking Digital Ecosystems and Technologies", IEEE signal processing conference, MEPCON "International Middle East Power Systems Conference", IEEE conference on communication, IEECON "International Electrical Engineering Congress", and KES2014 "International Conference on KnowledgeBased and Intelligent Information \& Engineering Systems". Same as the conferences, table 4 shows the distribution of papers over the journals. Obviously, we can see that the IJCA "International Journal of Computer Application" were had the majority published papers with $12.5 \%$. While each of the other papers was published in different journal with $6.25 \%$ which are IJAERT "International Journal of Advanced Engineering Research and Technology", IJETAE "International Journal of Emerging Technology and Advanced Engineering", IJASEIT "International Journal on Advanced Science, Engineering and Information Technology", IJEEI "International Journal on Electrical Engineering and Informatics", IJSRP "International Journal of Scientific and Research Publication", IJARS "International Journal of Anatomy Radiology and Surgery", IJSSMET "International Journal of Service Science,
Management, Engineering, and Technology", IJAIA "International Journal of Artificial Intelligence \& Applications", INDJST "Indian Journal of Science and Technology", IJTPE "International Journal on Technical and Physical Problems of Engineering", CNSNS "Communications in Nonlinear Science and Numerical Simulation", ICA "International Control and Automation", ECM "Energy Conversion and Management", and ACISC "Applied Computational Intelligence and Soft Computing".

\begin{tabular}{|l|c|c|c|}
\hline \multicolumn{4}{|c|}{ TABLE 3 } \\
CONFERENCES \\
\hline $\begin{array}{c}\text { DISTRIBUTION OF PAPERS OVER THE } \\
\text { name }\end{array}$ & $\begin{array}{c}\text { No. } \\
\text { of } \\
\text { the } \\
\text { paper }\end{array}$ & Freq. & $\begin{array}{c}\text { Percentage } \\
(\%)\end{array}$ \\
\hline EDT & A22 & 1 & $16.67 \%$ \\
\hline $\begin{array}{c}\text { IEEE Signal } \\
\text { Processing }\end{array}$ & A9 & 1 & $16.67 \%$ \\
\hline MEPCON & A20 & 1 & $16.67 \%$ \\
\hline $\begin{array}{l}\text { IEEE } \\
\text { (International } \\
\text { conference on } \\
\text { communication) }\end{array}$ & A14 & 1 & $16.67 \%$ \\
\hline IEECON & A8 & 1 & $16.67 \%$ \\
\hline KES2014 & A15 & 1 & $16.67 \%$ \\
\hline
\end{tabular}

\begin{tabular}{|c|c|c|c|}
\hline \multicolumn{4}{|c|}{$\begin{array}{c}\text { TABLE } 4 \\
\text { DISTRIBUTION OF PAPERS OVER THE } \\
\text { JOURNALS }\end{array}$} \\
\hline $\begin{array}{c}\text { Journal } \\
\text { name }\end{array}$ & $\begin{array}{l}\text { No. of } \\
\text { the paper }\end{array}$ & Freq. & Percentage (\%) \\
\hline IJAERT & A1 & 1 & $6.25 \%$ \\
\hline IJCA & A2 A6 & 2 & $12.5 \%$ \\
\hline IJETAE & A3 & 1 & $6.25 \%$ \\
\hline IJASEIT & $\overline{\mathrm{A} 4}$ & 1 & $6.25 \%$ \\
\hline IJEEI & A7 & 1 & $6.25 \%$ \\
\hline IJSRP & A11 & 1 & $6.25 \%$ \\
\hline IJARS & A13 & 1 & $6.25 \%$ \\
\hline IJSSMET & A17 & 1 & $6.25 \%$ \\
\hline IJAIA & A18 & 1 & $6.25 \%$ \\
\hline INDJST & A19 & 1 & $6.25 \%$ \\
\hline IJTPE & A5 & 1 & $6.25 \%$ \\
\hline CNSNS & A10 & 1 & $6.25 \%$ \\
\hline ICA & A12 & 1 & $6.25 \%$ \\
\hline ECM & A16 & 1 & $6.25 \%$ \\
\hline ACISC & A21 & 1 & $6.25 \%$ \\
\hline
\end{tabular}

\subsection{RESEARCH QUESTION 1}

When we searched for the related research papers between the years 2010 and 2018, we found many meta-heuristic 
algorithms that were used in the PID gain tuning. Those algorithms are:

- Genetic Algorithm (GA)

- Bacterial Foraging Optimization Algorithm (BFOA)

- Artificial Bee Cloning algorithm (ABC)

- fruit fly optimization algorithm (FOA)

- Particle Swarm Optimization algorithm (PSO)

- Ant Colony Optimization algorithm (ACO)

- Firefly Algorithm (FA)

- Evolutionary Programming (EP)

The chart in Figure 2 illustrates the percentage of each algorithm used in the selected papers. We can infer from this chart that GA, PSO, and ACO have the highest percentage among the whole algorithms with a percentage of $71 \%$. Thus, it means that those techniques are the most applied techniques due to their high efficiency and fast performance. Then ABC algorithm comes next with $11 \%$. While the rest has an overall percentage of $29 \%$.

\section{Algorithms used in PID gain tuning}

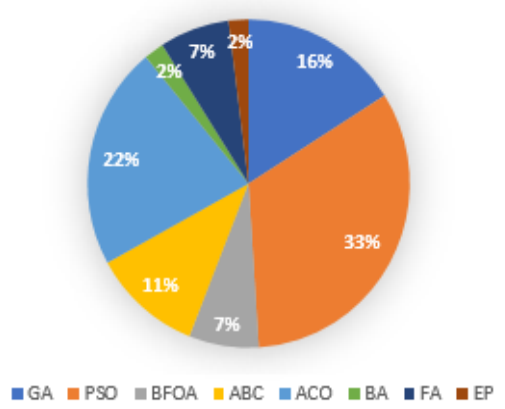

Figure 2- percentages of algorithms used in PID gain tuning

\subsection{RESEARCH QUESTION 2}

There are many domains that applied the optimization techniques in PID gain tuning. Five general domains were identified listed as follows:

- Mechanical Engineering

- Solar Energy

- Power System

- Magnetisms

- Industrial

As shown in the Figure 3, the most active domains used in PID tuning by optimization techniques were the power system and the mechanical engineering with a percentage of $36 \%$ and $32 \%$, respectively. The third active domain was the industrial with a percentage of $24 \%$. While the fourth and fifth were the magnetisms and the solar energy with a percentage of $8 \%$ among all domains.

For instance, in the power system domain, Power System Stabilizers (PSS) considered as an application that uses the optimization techniques in PID gain tuning. In order to damp the low frequency oscillations (LFO) in the power system,
PSS are used to produce signals of damping controls for the excitation system [26].

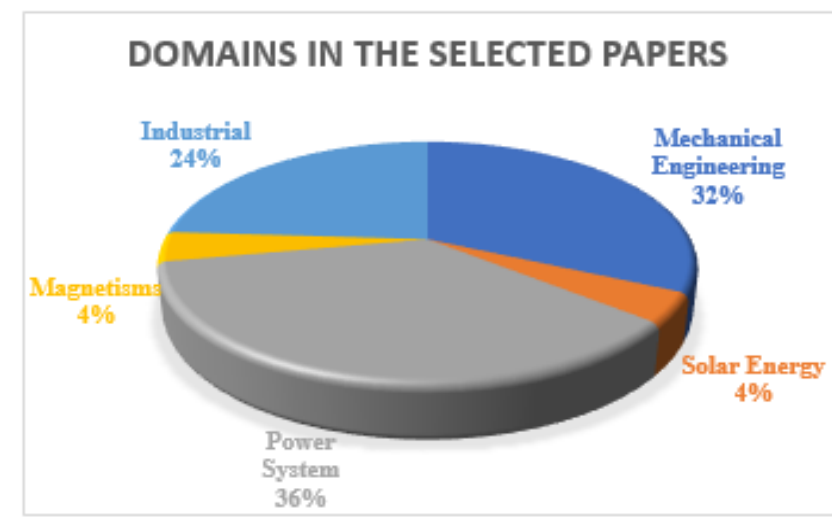

Figure 3- percentages of selected papers in each domain tuning.

\subsection{RESEARCH QUESTION 3}

Searching deeply in the selected research papers, we found that the PSO is the mostly used algorithms among all other algorithms proposed in the papers. Then ACO and the last one is GA. The Particle Swarm Optimization (PSO) is the mostly used due to many reasons: It gives the best result and the most efficient solutions, where it is applying a swarm of particles. Those particles are considered as candidates' solutions for PID parameters. Furthermore, the iterations in the PSO considered as memory where the information needed saved in the iterations, and that information will be used to find the best PID parameters values. In each of the iterations, a comparison between the initial solution and its neighbor is done to find and save the result as the best result found. Therefore, the personal position and the global positions are the target of the swarms' particles. Comparing to Genetic Algorithm (GA) and Ant Colony Optimization (ACO), the GA has many steps like selection, crossover, and mutation which considered as a long process compared to PSO. Thus, leads to slacken the whole process. Also, in genetic algorithms to reach to optimum solution we need to make recombination and mutation to the individuals to make or create new offspring, on other hand, in PSO the particles only move to the optimum (best) solution. Moreover, PSO has the superior over the ACO in PID tuning since its easy for the ACO to fall wrongly to the local optimal solution due to the lack of guiding process that leads to good solutions. Although, both GA and ACO have close results as both has some common features, for example, both have low convergence to the optimum solution. All these factors made the PSO better and more accurate and precise than the GA and ACO in PID

\section{LIMITATIONS OF THIS REVIEW}

In our study, only journals and conference papers are used in PID gain tuning. After applying the filtration process, many irrelevant articles were found from the large number of papers, and those irrelevant articles consisted the majority. Thus, compared to the papers found, a small number of papers 
were considered to confine the relevant papers needed in our study. Furthermore, only the optimization techniques which were suggested and used in more than one paper are considered in this review. Moreover, the quality assessment rules were applied to filter our search results which leads to synthesize those results.

\section{CONCLUSIONS AND FUTUTE WORK}

related to the PID gain tuning using the optimization techniques from 2010 and 2018. Where we answered three RQs based on the selected 22 papers. The conclusion of our review is as the following:

RQ1: The most used optimization techniques in PID controller gain tuning are $\mathrm{PSO}, \mathrm{GA}$, and $\mathrm{ACO}$.

RQ2: The most active domains used in PID tuning by optimization techniques were the power system and the mechanical engineering.

RQ3: The most effective optimization technique found compared to the techniques that are mostly used in the selected papers is the PSO.

Further future work can include:

1. An extended timeline, including more papers into the review.

2. Raise more research questions and providing more information regarding the topic.

3. A look at other methods of tuning PID controllers such as:
a. Fuzzy Logic Techniques
b. Artificial Neural Networks
c. Ziegler-Nicholas method

\section{REFERENCES}

[1] K. H. Ang, G. Chong, S. Member, and Y. Li, "PID Control System Analysis , Design , and Technology," vol. 13, no. 4, pp. 559-576, 2005.

[2] J. C. Basilio, "Design of PI and PID Controllers With Transient Performance Specification," no. December 2002, 2014

[3] H. O. Bansal, R. Sharma, and P. R. Shreeraman, "PID Controller Tuning Techniques : A Review," no. November 2012, 2017.

[4] S. Ghosal, R. Darbar, and B. Neogi, "Application of Swarm Intelligence Computation Techniques in PID Controller Tuning : A Review Application of Swarm Intelligence Computation Techniques in PID Controller Tuning: A Review," no. January 2015, 2012.

[5] V. Singh and V. K. Garg, "Tuning of PID Controller for Speed Control of DC Motor Using Soft Computing Techniques - A Review," vol. 9, no. 9, pp. 1141-1148, 2014.

[6] P. Cominos and N. Munro, "PID controllers: recent tuning methods and design to specification," vol. 149, no. I, 2002.

[7] B. Kitchenham, and S. Charters, "Guidelines for performing Systematic Literature Reviews in Software Engineering", EBSE Technical Report, vol. 01, no. 4ve, 2007.

[8] A. Nayak, M. Singh, and M. E. Scholar, "Research Paper STUDY OF TUNING OF PID CONTROLLER BY USING PARTICLE SWARM OPTIMIZATION," 2015.

[9] S. M. Girirajkumar, "Tuning of a PID Controller for a Real Time Industrial Process using Particle Swarm Optimization,” pp. 35-40, 2010.

[10] R. Bindu and M. K. Namboothiripad, "Tuning of PID Controller for DC Servo Motor using Genetic Algorithm," vol. 2, no. 3, 2012.

[11] M. I. Solihin, L. F. Tack, and M. L. Kean, "Tuning of PID
Controller Using Particle Swarm Optimization (PSO )," 2011.

A. Jalilvand, A. Kimiyaghalam, A. Ashouri, and H. Kord, "OPTIMAL TUNING OF PID CONTROLLER PARAMETERS ON A DC MOTOR BASED ON ADVANCED PARTICLE SWARM OPTIMIZATION," no. December, pp. 10-17, 2011.

[13] S. M. Girirajkumar, "PSO based Tuning of a PID Controller for a High Performance Drilling Machine," vol. 1, no. 19, pp. 12-18, 2010

[14] M. Omar, M. Soliman, A. M. A. Ghany, and F. Bendary, "Optimal Tuning of PID Controllers for Hydrothermal Load Frequency Control Using Ant Colony Optimization," vol. 5, no. 3, pp. 348360, 2013.

[15] "Particle Swam Optimization based Optimal PID Parameters for Air Heater Temperature Control System.pdf."

[16] S. Sahlan, "Particle Swarm Optimization for Multivariable PID Controller Tuning," no. July 2018, 2013.

[17] W. Chang and S. Shih, "Commun Nonlinear Sci Numer Simulat PID controller design of nonlinear systems using an improved particle swarm optimization approach," Commun. Nonlinear Sci. Numer. Simul., vol. 15, no. 11, pp. 3632-3639, 2010.

[18] N. S. Rathore, N. Kundariya, and A. Narain, "PID Controller Tuning in Reverse Osmosis System based on Particle Swarm Optimization," vol. 3, no. 6, pp. 1-5, 2013

[19] I. Control and A. A. Aly, "PID Parameters Optimization Using Genetic Algorithm Technique for Electrohydraulic Servo Control System," no. January 2011, 2014.

[20] R. Paper, A. Y. Jaen-cuellar, L. Morales-velazquez, and R. A. Osornio-rios, "PID-Controller Tuning Optimization with Genetic Algorithms in Servo Systems," 2013.

[21] B. Nagaraj, P. Vijayakumar, and E. Pro, "A C OMPARATIVE S TUDY OF PID C ONTROLLER T UNING,” vol. 5, 2011.

[22] "Ant colony optimization algorithm applied to ship steering control.pdf."

[23] H. Fang, L. Chen, and Z. Shen, "Application of an improved PSO algorithm to optimal tuning of PID gains for water turbine governor," Energy Convers. Manag., vol. 52, no. 4, pp. 1763-1770, 2011 .

[24] J. Kaliannan, A. Baskaran, and N. Dey, "Automatic Generation Control of Thermal-Thermal-Hydro Power Systems with PID Controller using Ant Colony Optimization," no. September, 2015.

[25] S. J. Bassi, M. K. Mishra, and E. E. Omizegba, "AUTOMATIC TUNING OF PROPORTIONAL - INTEGRAL - DERIVATIVE ( PID ) CONTROLLER USING PARTICLE SWARM OPTIMIZATION ( PSO ) ALGORITHM," vol. 2, no. 4, pp. 25$34,2011$.

[26] "Optimal PID power system stabilizer tuning based on particle swarm optimization," vol. 4, no. 4, pp. 379-383, 2011.

[27] G. Shabib, M. A. Gayed, and A. M. Rashwan, "Optimal Tuning of PID Controller for AVR System using Modified Particle Swarm Optimization," pp. 305-310, 2010.

[28] I. Chiha, N. Liouane, and P. Borne, "Ant Colony Optimization," vol. 2012, no. 1, 2012.

[29] H. Zhang, Y. Cai, and Y. Chen, "Parameter Optimization of PID Controllers Based on Genetic Algorithm," pp. 47-49.

[30] M. Azzeh, A. B. Nassif, S. Banitaan, and F. Almasalha, "Pareto efficient multi-objective optimization for local tuning of analogybased estimation," Neural Comput. Appl., vol. 27, no. 8, pp. 2241$2265,2016$.

[31] A. B. Nassif, A. Elnagar, I. Shahin, and S. Henno, "Deep learning for Arabic subjective sentiment analysis: Challenges and research opportunities," Applied Soft Computing, vol. 98. Elsevier Ltd, p. 106836,2020

Authors Contributions: Abdallah Abushawish and Mohammed Hamadeh wrote the paper. Ali Bou Nassif conducted the methodology and revised the paper

\section{Creative Commons Attribution License 4.0 (Attribution 4.0 International, CC BY 4.0)}

This article is published under the terms of the Creative Commons Attribution License 4.0

https://creativecommons.org/licenses/by/4.0/deed.en_US 


\section{APPENDICES}

Appendix A

Table 1

\begin{tabular}{|c|c|c|}
\hline A1 & Study of tuning of PID controller by using particle swarm optimization & [8] \\
\hline A2 & Tuning of a PID controller for a real time industrial process using particle swarm optimization & [9] \\
\hline A3 & Tuning of PID controller for DC servo motor using genetic algorithm & {$[10]$} \\
\hline A4 & Tuning of PID controller using particle swarm optimization (PSO) & {$[11]$} \\
\hline A5 & $\begin{array}{c}\text { Optimal tuning of PID controller parameters on a DC motor based on advanced particle swarm optimization } \\
\text { algorithm }\end{array}$ & [12] \\
\hline A6 & PSO based tuning of a PID controller for a high-performance drilling machine & [13] \\
\hline A7 & Optimal tuning of PID controllers for hydrothermal load frequency control using ant colony optimization & {$[14]$} \\
\hline A8 & Particle swam optimization based optimal PID parameters for air heater temperature control system & [15] \\
\hline A9 & $\begin{array}{l}\text { Particle swarm optimization for multivariable PID controller tuning } \\
\end{array}$ & {$[16]$} \\
\hline A10 & PID controller design of nonlinear systems using an improved particle swarm optimization approach & {$[17]$} \\
\hline A11 & PID controller tuning in reverse osmosis system based on particle swarm optimization & [18] \\
\hline A12 & PID parameters optimization using genetic algorithm technique for electrohydraulic servo control system & {$[19]$} \\
\hline A13 & PID-controller tuning optimization with genetic algorithms in servo systems & {$[20]$} \\
\hline A14 & A comparative study of PID controller tuning using GA, EP, PSO and ACO & {$[21]$} \\
\hline A15 & Ant colony optimization algorithm applied to ship steering control & {$[22]$} \\
\hline A16 & Application of an improved PSO algorithm to optimal tuning of PID gains for water turbine governor & {$[23]$} \\
\hline A17 & $\begin{array}{l}\text { Automatic generation control of Thermal-Thermal-Hydro power systems with PID controller using ant colony } \\
\text { optimization }\end{array}$ & {$[24]$} \\
\hline A18 & $\begin{array}{l}\text { Automatic tuning of proportional-integral-derivative (PID) controller using particle swarm optimization (PSO) } \\
\text { algorithm }\end{array}$ & {$[25]$} \\
\hline A19 & Optimal PID power system stabilizer tuning based on particle swarm optimization & [26] \\
\hline A20 & Optimal tuning of PID controller for AVR system using modified particle swarm optimization & {$[27]$} \\
\hline A21 & Tuning PID controller using multi-objective ant colony optimization & {$[28]$} \\
\hline A22 & Parameter optimization of PID controllers based on genetic algorithm & [29] \\
\hline
\end{tabular}


Table 2

\begin{tabular}{|c|c|c|c|c|c|c|c|}
\hline ID & QAR1 & QAR2 & QAR3 & QAR4 & QAR5 & QAR6 & Total \\
\hline A1 & 1 & 0.75 & 1 & 1 & 0.75 & 0.75 & 5.25 \\
\hline A2 & 0.75 & 0.25 & 1 & 0.5 & 0.75 & 0.75 & 4 \\
\hline A3 & 1 & 0.5 & 1 & 0.75 & 0.5 & 0.5 & 4.25 \\
\hline A4 & 1 & 1 & 0.75 & 1 & 0.75 & 0.75 & 5.25 \\
\hline A5 & 1 & 1 & 1 & 1 & 0.75 & 1 & 5.75 \\
\hline A6 & 1 & 0.5 & 1 & 0.25 & 0.75 & 1 & 4.5 \\
\hline A7 & 1 & 0.75 & 0.75 & 0.25 & 1 & 1 & 4.75 \\
\hline A8 & 0.75 & 0.25 & 0.75 & 0.75 & 0.5 & 0.5 & 3.5 \\
\hline A9 & 1 & 1 & 1 & 0 & 0.75 & 0.75 & 4.5 \\
\hline A10 & 1 & 0.5 & 0.75 & 0 & 0.75 & 0.5 & 3.5 \\
\hline A11 & 1 & 1 & 1 & 0.5 & 1 & 1 & 5.5 \\
\hline A12 & 1 & 1 & 0.75 & 0.25 & 1 & 0.75 & 4.75 \\
\hline A13 & 1 & 1 & 1 & 0.5 & 1 & 1 & 5.5 \\
\hline A14 & 1 & 1 & 1 & 1 & 1 & 1 & 6 \\
\hline A15 & 1 & 0.5 & 1 & 1 & 0.75 & 0.75 & 5 \\
\hline A16 & 0.75 & 0.5 & 0.75 & 0.25 & 0.75 & 0.5 & 3.5 \\
\hline A17 & 1 & 1 & 0.5 & 0.75 & 1 & 1 & 5.25 \\
\hline A18 & 1 & 1 & 1 & 1 & 1 & 1 & 6 \\
\hline A19 & 0.75 & 0.75 & 1 & 0.5 & 1 & 0.5 & 4 \\
\hline A20 & 1 & 0.75 & 1 & 1 & 0.5 & 0.5 & 4.75 \\
\hline A21 & 1 & 0.75 & 1 & 0.75 & 0.75 & 0.75 & 5 \\
\hline A22 & 1 & 1 & 1 & 0.75 & 1 & 1 & 5.75 \\
\hline
\end{tabular}

